International Journal of Pure and Applied Mathematics

Volume 85 No. 2 2013, 297-302

ISSN: 1311-8080 (printed version); ISSN: 1314-3395 (on-line version)

url: http://www.ijpam.eu

doi: http://dx.doi.org/10.12732/ijpam.v85i2.9

ijpam.eu

\title{
A CONTINUUM OF HAMILTONIAN STRUCTURES FOR THE TWO-DIMENSIONAL ISOTROPIC HARMONIC OSCILLATOR
}

\author{
Juan M. Romero ${ }^{1 \S}$, Adolfo Zamora ${ }^{2}$ \\ ${ }^{1,2}$ Department of Applied Mathematics and Systems \\ UAM-Cuajimalpa \\ Mexico, D.F. 01120, MEXICO
}

\begin{abstract}
We show the existence of a continuum of Hamiltonian structures for the two-dimensional isotropic harmonic oscillator. In particular, a continuum of Hamiltonian structures having noncommutative coordinates is presented. A study of the symmetries of these structures is performed and their physical plausibility is discussed.
\end{abstract}

AMS Subject Classification: 70H05, 70H15

Key Words: Hamilton's equations, symplectic structures, inverse problem

\section{Introduction}

It is well known that for some equations of motion there exist inequivalent Hamiltonian/Lagrangian structures. This is the so-called inverse problem in variational calculus [1]. It is also well known that these alternative structures yield different quantum theories $[2,3,4,5,6]$. In this work we present a continuum of Hamiltonian structures for the two-dimensional isotropic harmonic oscillator; in particular, a continuum of Hamiltonian structures with noncommutative coordinates. We also perform a study of their symmetries.

Received: November 24, 2012

(c) 2013 Academic Publications, Ltd.

${ }^{\S}$ Correspondence author url: www.acadpubl.eu 


\section{The Two-Dimensional Isotropic Harmonic Oscillator}

To begin with, let us recall that the usual Hamiltonian structure for the twodimensional isotropic harmonic oscillator is given by the Hamiltonian

$$
H_{0}=\frac{1}{2 m}\left(p_{x}^{2}+p_{y}^{2}\right)+\frac{m \omega^{2}}{2}\left(x^{2}+y^{2}\right),
$$

with Poisson brackets

$$
\left\{x, p_{x}\right\}_{0}=1, \quad\left\{y, p_{y}\right\}_{0}=1, \quad \text { zero otherwise. }
$$

The rotational invariance is an important symmetry of the isotropic harmonic oscillator. This symmetry is compatible with the usual Hamiltonian structure as $H_{0}$ and the Poisson brackets from Eq. (2.2) are both invariant under rotations

$$
\begin{aligned}
& x^{\prime}=x \cos \theta-y \sin \theta, \\
& y^{\prime}=x \sin \theta+y \cos \theta .
\end{aligned}
$$

Observe that this symmetry has $r^{2}=x^{2}+y^{2}$ as an invariant. Another property physically observable is that in the limit $\omega \rightarrow 0$ the oscillator becomes a free particle. Clearly the usual Hamiltonian formulation above is also compatible with this.

Now, let us consider the Hamiltonian structure given by the Hamiltonian

$$
\begin{aligned}
H_{\alpha} & =\left[\frac{1}{2 m}\left(p_{y}^{2}-p_{x}^{2}\right)+\frac{m \omega^{2}}{2}\left(y^{2}-x^{2}\right)\right] \sin \alpha \\
& -\omega\left[x p_{y}-y p_{x}\right] \cos \alpha
\end{aligned}
$$

with Poisson brackets

$$
\begin{array}{ll}
\{x, y\}_{\alpha}=\cos \alpha / m \omega, & \left\{x, p_{x}\right\}_{\alpha}=-\sin \alpha, \\
\left\{y, p_{y}\right\}_{\alpha}=\sin \alpha, & \left\{p_{x}, p_{y}\right\}_{\alpha}=m \omega \cos \alpha,
\end{array}
$$

and zero otherwise. From a straightforward calculation it can be shown that for every $\alpha$ the equations of motion are

$$
\begin{array}{ll}
\dot{x}=p_{x} / m=\left\{x, H_{\alpha}\right\}_{\alpha}, \quad \dot{p}_{x}=-m \omega^{2} x=\left\{p_{x}, H_{\alpha}\right\}_{\alpha}, \\
\dot{y}=p_{y} / m=\left\{y, H_{\alpha}\right\}_{\alpha}, \quad \dot{p}_{y}=-m \omega^{2} y=\left\{p_{y}, H_{\alpha}\right\}_{\alpha},
\end{array}
$$

i.e., this is a continuum of Hamiltonian structures for the two-dimensional isotropic harmonic oscillator. The cases $\alpha=\pi / 2$ and $\alpha=\pi$ were recently 
reported in Ref. [7]. Note that for $\alpha \neq(2 n+1) \pi / 2$, with $n$ an integer, after quantizing the system we will have a noncommutative space in the coordinates.

It can be shown that also the Hamiltonian structure given by the Hamiltonian

$$
\begin{aligned}
H_{\beta} & =\left[\frac{1}{2 m}\left(p_{y}^{2}-p_{x}^{2}\right)+\frac{m \omega^{2}}{2}\left(y^{2}-x^{2}\right)\right] \sin \beta \\
& +\left[\frac{p_{x} p_{y}}{m}+m \omega^{2} x y\right] \cos \beta
\end{aligned}
$$

with Poisson brackets

$$
\begin{aligned}
& \left\{x, p_{x}\right\}_{\beta}=-\sin \beta, \quad\left\{x, p_{y}\right\}_{\beta}=\cos \beta, \\
& \left\{y, p_{x}\right\}_{\beta}=\cos \beta, \quad\left\{y, p_{y}\right\}_{\beta}=\sin \beta,
\end{aligned}
$$

and zero otherwise, yields the equation of motion (2.6) for each $\beta$. The case $\beta=0$ was also reported in Ref. [7] (see also Ref. [1]).

Another Hamiltonian structure leading to the same equations of motion (2.6) is formed by the Hamiltonian

$$
\begin{aligned}
H_{\gamma} & =-\left[\frac{1}{2 m}\left(p_{y}^{2}+p_{x}^{2}\right)+\frac{m \omega^{2}}{2}\left(y^{2}+x^{2}\right)\right] \sinh \gamma \\
& +\left[\frac{p_{x} p_{y}}{m}+m \omega^{2} x y\right] \cosh \gamma,
\end{aligned}
$$

with Poisson brackets

$$
\begin{aligned}
& \left\{x, p_{x}\right\}_{\gamma}=\sinh \gamma, \quad\left\{x, p_{y}\right\}_{\gamma}=\cosh \gamma \\
& \left\{y, p_{x}\right\}_{\gamma}=\cosh \gamma, \quad\left\{y, p_{y}\right\}_{\gamma}=\sinh \gamma
\end{aligned}
$$

and zero otherwise. Once again, this structure yields the equations of motion (2.6) for every $\gamma$.

Now, for each $\alpha, \beta$ and $\gamma$, the quantities

$$
\begin{aligned}
D & =\left(\frac{p_{x} p_{y}}{m}+m \omega^{2} x y\right) \\
L & =x p_{y}-y p_{x}, \\
H_{ \pm} & =\frac{1}{2 m}\left(p_{y}^{2} \pm p_{x}^{2}\right)+\frac{m \omega^{2}}{2}\left(y^{2} \pm x^{2}\right) .
\end{aligned}
$$

are conserved. It can be seen that they are, in fact, the symmetry generators. For each $\alpha$, the algebra of these generators is

$$
\{D, L\}_{\alpha}=2 H_{+} \sin \alpha,
$$




$$
\begin{aligned}
\left\{D, H_{ \pm}\right\}_{\alpha} & =2 \omega H_{\mp} \cos \alpha+(1 \mp 1) \omega^{2} L \sin \alpha, \\
\left\{L, H_{ \pm}\right\}_{\alpha} & =-(1 \pm 1) D \sin \alpha, \\
\left\{H_{+}, H_{-}\right\}_{\alpha} & =2 \omega D \cos \alpha,
\end{aligned}
$$

whereas for each $\beta$,

$$
\begin{aligned}
\{D, L\}_{\beta} & =2 H_{+} \sin \beta \\
\left\{D, H_{ \pm}\right\}_{\beta} & =(1 \pm 1) \omega^{2} L \sin \beta \\
\left\{L, H_{ \pm}\right\}_{\beta} & =2 H_{\mp} \cos \beta-(1 \pm 1) D \sin \beta \\
\left\{H_{+}, H_{-}\right\}_{\beta} & =-2 \omega^{2} L \cos \beta
\end{aligned}
$$

and for each $\gamma$,

$$
\begin{aligned}
\{D, L\}_{\gamma} & =-2 H_{-} \sinh \gamma \\
\left\{D, H_{ \pm}\right\}_{\gamma} & =(1 \mp 1) \omega^{2} L \sinh \gamma \\
\left\{L, H_{ \pm}\right\}_{\gamma} & =2 H_{\mp} \cosh \gamma-(1 \mp 1) D \sinh \gamma \\
\left\{H_{+}, H_{-}\right\}_{\gamma} & =2 \omega^{2} L \cosh \gamma .
\end{aligned}
$$

Notice that as the Lie algebra of the constants of motion depends on the values of $\alpha, \beta$ and $\gamma$; for each of these values we will have a different symmetry group. As an example, for $\alpha=0$ the Hamiltonian $H_{\alpha=0}$ and its corresponding Poisson brackets are invariant under the rotations defined by Eq. (2.3) and also under the scaling transformations

$$
x^{\prime}=e^{\lambda} x, \quad y^{\prime}=e^{-\lambda} y,
$$

which are particularly interesting because they mix long and short scales together. The rotations are generated by $H_{+} / \omega$ and the scaling symmetry of Eq. (2.26) by $-D / \omega$. Observe that once $\alpha \neq 0$, the rotational invariance is lost. The Hamiltonian structures labeled $\beta$ and $\gamma$ are not rotationally invariant either. However, in the case $\beta=\gamma=0$, the structure is invariant under the scaling transformations from Eq. (2.26) which are generated by $L$. In the case $\beta=\pi / 2$, the Hamiltonian structure is invariant under the transformations

$$
\begin{aligned}
& x^{\prime}=x \cosh \theta+y \sinh \theta, \\
& y^{\prime}=x \sinh \theta+y \cosh \theta .
\end{aligned}
$$

That is, this structure posses the Lorentz symmetry. For this, the generator of the symmetry is $L$. Notice here that the invariant associated to these transformations is $s^{2}=x^{2}-y^{2}$. On the other hand, it can be shown that the generators 
of rotations for $\beta=0$ and $\beta=\pi$ are $L_{1}=x p_{x}-y p_{y}$ and $L_{2}=x p_{y}+y p_{x}$ respectively. Both of these are not conserved quantities; which confirms the fact that both systems have no rotational symmetry. The alternative Hamiltonian structures here presented have particular symmetries and, in this sense, they are all different.

\section{Conclusions}

We showed the existence of a continuum of Hamiltonian structures for the two-dimensional isotropic harmonic oscillator and a study of the symmetries of these structures was performed. In general, when the equations of motion of a physical system can be obtained from alternative Hamiltonian structures, it is difficult to argue why one chooses the standard structure over the others [3, $5,6]$. For the two-dimensional isotropic harmonic oscillator and the alternative structures here presented, this is not so complicated. As explained above, the case $\alpha=0$ can be clearly discarded because both $H_{\alpha=0}$ and its corresponding Poisson brackets lose meaning in the limit $\omega \rightarrow 0$. The other cases for $\alpha, \beta$ and $\gamma$ can, in principle, be discarded because they have no rotational symmetry. Thus, the best mathematical representation for this physical system is provided by the usual Hamiltonian structure. This, however, does not mean one should fully neglect the others as they may give a better representation of other systems and, in addition, may have interesting properties. For instance, in the Hamiltonian structure labeled $\alpha$ above, if $\cos \alpha \neq 0$ we will have noncommutative spaces. That is, we will have the commutation rules

$$
[\hat{x}, \hat{y}]=i \hbar \Theta, \quad \Theta=\frac{\cos \alpha}{m \omega} .
$$

Moreover, if $\cos \alpha>0$, it can be shown that with these commutation rules, the quantity $\hat{A}=\pi \hat{r}^{2}=\pi\left(\hat{x}^{2}+\hat{y}^{2}\right)$, has the spectrum $[8,9]$,

$$
A_{N}=2 \pi \hbar \Theta(N+1 / 2), \quad N=0,1,2, \ldots
$$

i.e. the area is quantized. Some authors [10] believe that the area of the horizon of events of a black hole has a spectrum of the form of Eq. (3.2). The Hamiltonian structures invariant under the scaling transformations from Eq. (2.26) are also interesting because they mix different scales together. It is also notable that the structure with $\beta=\pi / 2$ posses the Lorentz symmetry despite it is a non relativistic model. 


\section{References}

[1] R.M. Santilli, Foundations of Theoretical Mechanics. The Inverse Problem in Newtonian Mechanics: Part I, Springer-Verlag, New York (1978).

[2] E.P. Wigner, Do the equations of motion determine the quantum mechanical commutation relations?, Physical Review, 77 (1950), 711-712.

[3] S. Okubo, Does the equation of motion determine commutation relations?, Physical Review D, 22 (1980), 919-923.

[4] M. Henneaux, L.C. Shepley, Lagrangians for spherically symmetric potentials, Journal of Mathematical Physics, 23 (1982), 2101-2107.

[5] N.A. Lemos, Physical consequences of the choice of the Lagrangian, Physical Review D, 24 (1981), 1036-1039.

[6] S.A. Hojman, L.F. Urrutia, Comments on "Physical consequences of the choice of the Lagrangian", Physical Review D, 26 (1982), 527-528.

[7] M. Montesinos, G.F. Torres del Castillo, Symplectic quantization, inequivalent quantum theories, and Heisenberg's principle of uncertainty, Physical Review A, 70 (2004), 032104.

[8] J.M. Romero, J.A. Santiago, J.D. Vergara, A note about the quantum of area in a non-commutative space, Physical Review D, 68 (2003), 067503.

[9] S. Sivasubramanian, G. Srivastava, A. Vitiello, Y.N. Widom, Quantum dissipation induced noncommutative geometry, Physics Letters A, 311 (2003), 97-105.

[10] J.D. Bekenstein, Quantum black holes as atoms, gr-qc/9710076; A. Alekseev, A.P. Polychronakos, M. Smedbäck, On area and entropy of a black hole, Physics Letters B, 574 (2003), 296-300. 